Ann. Biol. anim. Bioch. Biophys., I974, 14 (4-B), 78I-79I.

\title{
EFFET LIPOLYTIQUE IN VIVO DE L'HORMONE SOMATOTROPE CHEZ DES AGNEAUX NORMAUX ET HYPOPHYSECTOMISÉS SOUMIS OU NON A UN JEÛNE
}

\author{
A. VÉZINHET, J.-P. KHÉRIF et E. BOUTHIER \\ Station de Physiologie animale, \\ École nationale supérieure agronomique, $I, N . R . A$, \\ 34060 Montpellier Cedex
}

\section{RÉSUMÉ,}

L'effet lipolytique d'un traitement aigu à l'hormone somatotrope bovine (BGH) chez l'Agneau normal et hypophysectomisé est étudié pour des animaux nourris ad libitum ou après un jeâne solide de 24 heures. L'évolution de la glycémie est suivie dans les mêmes conditions.

- La lipolyse induite par la BGH lorsque les agneaux sont nourris ad libitum semble être plus intense chez les agneaux normaux que chez les hypophysectomisés.

- Les taux plasmatiques en acides gras libres après 24 heures de jeûne sont plus faibles chez les hypophysectomisés que chez les normaux.

- Ces deux observations laissent penser que chez les agneaux hypophysectomisés le maintien des réserves adipeuses est assuré plus efficacement que chez les normaux.

- Par contre dans le cas des agneaux à jeun la réponse lipolytique à la BGH est particulièrement intense chez les hypophysectomisés.

- L'abaissement transitoire des taux d'acides gras libres 30 minutes après un traitement $\mathrm{BGH}$ ne semble pas pouvoir être mis en relation avec un effet propre de l'hormone.

- Les variations de la glycémie sont peu importantes après un traitement à la BGH, quelles que soient les conditions alimentaires dans lesquelles se trouve l'animal.

\section{INTRODUCTION}

Dans un précédent travail VézINHET (I973), nous avons montré que l'hypophysectomie conduit à une accumulation des lipides de réserves dans les carcasses de mouton, tandis qu'un traitement chronique à l'hormone somatotrope (GH) réduit considérablement ce phénomène. CHRÉTIEN et al. (I973) ont signalé que l'hypophysectomie chez le Rat diminue la mobilisation des graisses en réponse à la fois à un jeûne prolongé ou à une stimulation hormonale. On peut donc admettre que la GH est susceptible de jouer un rôle dans la constitution et l'utilisation des graisses de réserve. Plusieurs auteurs ont suggéré que le métabolisme des lipides, en particulier le phénomène de lipolyse était influencé par l'hormone de croissance. Depuis RABEN et Hollenberg (I958) travaillant chez le Chien, on sait que la GH influence la libé- 
ration des acides gras libres. Ce rôle a été confirmé in vivo pour d'autres espèces. Chez les ovins il existe peu de travaux, cependant Manss et BoDa (I965), MAChLIN (I968) ont signalé que l'injection de GH ovine provoquait chez des moutons une élévation du taux plasmatique en acides gras non estérifiés. D'autre partles taux plasmatiques en GH ont été étudiés chez les bovins et les ovins notamment par TRENKLE et IRVIN (I97I), Trenkle (I97I). Chez les ovins Charrier (I973) a mesuré entre le cent cinquième jour de vie fotale et le deux cent cinquantième jour de vie postnatale l'évolution des concentrations hypophysaires en GH.

Nous nous proposons ici de suivre l'évolution de certains métabolites circulants chez des agneatux hypophysectomisés, traités ou non à la GH après avoir été soumis à un jeûne ou nourris ad libitum. Nous avons réalisé parallèlement des déterminations d'acides gras libres plasmatiques et de glycémie. On sait en effet que chez les monogastriques les interrelations entre les métabolismes des sucres et des lipides sont étroites.

Ce travail devrait permettre de préciser le rôle joué par la $\mathrm{GH}$ et l'état nutritionnel dans le métabolisme énergétique des ovins en même temps qu'il pourrait nous éclairer sur l'intervention des hormones dans les variations de composition corporelles en réserves adipeuses.

\section{MATÉRIEI， ET MÉTHODES}

Quarante-neuf agneaux mâles Mérinos d'Avles élevés au laboratoire ont été utilisés. Trente et un d'entre eux étaient hypophysectomisés, les 18 autres étaient normaux. Les hypophysectomies étaient pratiquées à l'âge de 50 jours par voie paraphyngienne, transphénoïdale. Les animaux étaient maintenus en survie dans des conditions précédemment décrites (VEzINHET, I968). Vingt jours après l'intervention, les agneaux ayant récupéré du choc opératoire, nous les avons traités à l'hormone somatotrope bovine $(\mathrm{BGH})$ après un jeûne solide de $\mathbf{2 4}$ heures qui se continuait pendant toute la durée des prélèvements, soit 24 heures supplémentaires. Dix jours plus tard, I 5 d'entre eux étaient à nouveau soumis au même jeûne, cette fois sans traitement hormonal. Les 16 autres hypophysectomisés, nourris ad libitum, recevaient ou non de la BGH. Pour les I 8 agneaux normaux utilisés aux mêmes âges que les hypophysectomisés, un premier lot de Io agneaux a été traité à la BGH après un jeûne de 24 heures puis Io jours plus tard avec un régime ad libitum. Les huit autres placés dans les mêmes conditions alimentaires que les précédents ne recevaient pas de BGH.

La BGH (Byla lot or-69, Activité I, I UI par mg) était injectée le matin par voie intraveineuse à la dose de I $\mathrm{mg}$ par $\mathrm{kg}$ de poids vif. Les échantillons de sang étaient prélevés par ponction intracardiaque au temps o précédant le traitement puis 30 minutes, I, 2, 3, 5, 8 et 24 heures après celui-ci. Les témoins recevaient une injection de sérum physiologique ( $\mathrm{NaCl} 9 \mathrm{p}$. I ooo).

Les mesures des concentrations plasmatiques en glucides réducteurs étaient réalisées par la technique de Nelson (I944) et celles des acides gras libres par la méthode de Dole et MeinerTz (1960).

L'analyse des résultats a consisté en une simple comparaison entre les moyennes des différents lots expérimentaux. En raison des corrélations qui existent entre les valeurs obtenues à des temps successifs, aucune analyse statistique rigoureuse n'a pu être mis en place dans le cadre de cette étude.

\section{RÉSULTATS}

Le tableau I montre les gains de poids réalisés par des agneaux normaux et par des hypophysectomisés dans les 30 jours après l'opération. On constate que 20 jours après l'intervention les animaux ont récupéré de la perte de poids due au choc 
opératoire. On peut donc estimer que leur utilisation à partir de ce moment-là pour des études métaboliques est justifiée et que les différences qui seront observées par rapport aux animaux normaux résulteront bien de leur état d'hypophysoprive.

\section{TABI.EAU I}

Comparaison entre les évolutions pondérales d'agneaux normaux et d'agneaux hypophysectomisés à l'âge de 50 jours et maintenus en survie jusqu'à l'âge de 80 jours

\begin{tabular}{|c|c|c|}
\hline \multirow{2}{*}{ Age en jours } & \multicolumn{2}{|c|}{ Poids vif en $\mathrm{kg}\rfloor$ erreur type } \\
\hline & $\begin{array}{c}\text { Agneaux hypophysectomisés } \\
n=31\end{array}$ & $\begin{array}{c}\text { Agneaux normaux } \\
n=18\end{array}$ \\
\hline 50 & $16,0 \pm 0,23$ & $16,3 \pm 0,4^{\prime} \mathrm{t}$ \\
\hline 55 & $15,6=0,31$ & - \\
\hline 60 & $15,5 \pm 0,3$ & $17,7 \pm 0,63$ \\
\hline 65 & $15,3 \pm 0,32$ & 工 \\
\hline 70 & $16,0 \pm 0,3$ & $19,9 \pm 0,77$ \\
\hline 75 & $16,2 \pm 0,3 / \mathbf{t}$ & - \\
\hline 80 & $16,3 \pm 0,34$ & $22,1 \pm 0,9^{\prime}$ \\
\hline
\end{tabular}

Acides gras libres (tabl. 2)

Du tableau 2 il ressort que pour des agneaux normaux et hypophysectomisés nourris ad libitum, on n'observe pas de variation notable de la teneur en acides gras libres plasmatiques, sur des prélèvements effectués pendant 24 heures après une injection de serum physiologique. Par contre si des animaux semblables subissent un traitement aigu à la $\mathrm{BGH}$, on note une élévation des acides gras libres. Pour les agneaux normaux cette augmentation est nettement marquée dès la première heure après le traitement avec un maximum se situant à la cinquième heure. Au temps 24 heures la valeur observée est du même ordre que celle du temps o précédant l'injection de $\mathrm{BGH}$.

Pour les agneaux hypophysectomisés le même phénomène est observé avec une amplitude moindre. Il faut attendre la cinquième heure après le traitement pour avoir une différence nette avec le temps o. La valeur maximale est obtenue seulement à 8 heures. Comme pour les normaux, au temps 24 heures, le taux en acides gras libres du plasma est voisin de celui du temps o.

\section{Glycémie (tabl. 3)}

Pour des animaux nourris ad libitum, dans tous les cas la glycémie des agneaux hypophysectomisés est nettement inférieure (environ $30 \mathrm{p}$. I00) à celle des normaux. Le traitement à la BGH semble n'avoir un effet que chez les hypophysectomisés pour lesquels une hypopglycémie est observée à la première heure après le traitement. Cette hypoglycémie est corrigée dès la troisième heure. 
A. VÉZINHET, J.-P. KHÉ,RIF, E. BOUTHIER

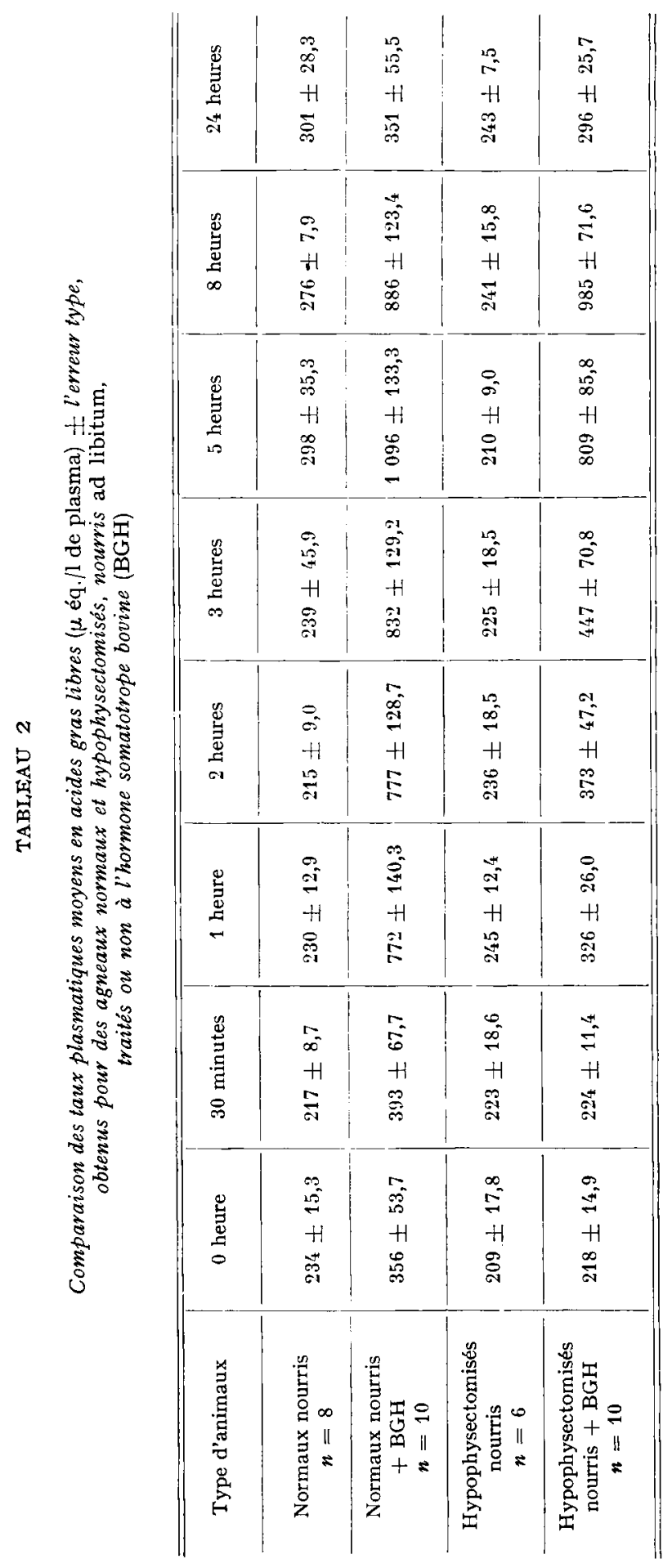


BGH E'T LIPOLYSE CHEZ L'AGNEAU

\begin{tabular}{|c|c|c|c|c|c|}
\hline \multirow{9}{*}{ 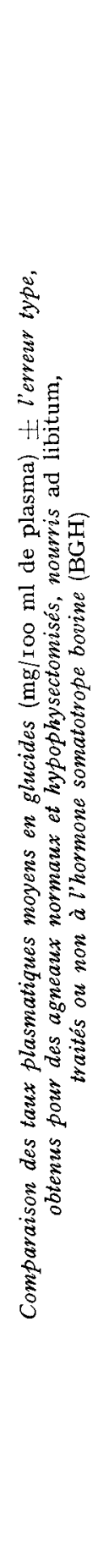 } & 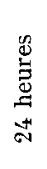 & $\begin{array}{l}m \\
\stackrel{-}{=} \\
H \\
\stackrel{+}{g} \\
\stackrel{8}{=}\end{array}$ & $\begin{array}{l}\infty \\
0 \\
\text { ô } \\
\text { H } \\
H \\
0 \\
0 \\
0 \\
0\end{array}$ & 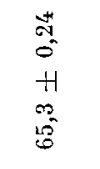 & $\begin{array}{l}\stackrel{0}{0} \\
\stackrel{+}{+1} \\
\frac{8}{8}\end{array}$ \\
\hline & 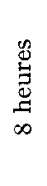 & 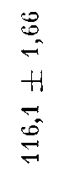 & $\begin{array}{l}a \\
\infty \\
\infty \\
\infty \\
H \\
- \\
a \\
0\end{array}$ & 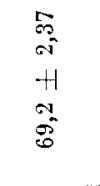 & $\begin{array}{l}\stackrel{g}{+} \\
\stackrel{-}{+} \\
0 \\
0 \\
50\end{array}$ \\
\hline & 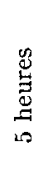 & 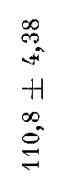 & $\begin{array}{l}\frac{\infty}{2} \\
+1 \\
\infty \\
\infty \\
9 \\
9\end{array}$ & 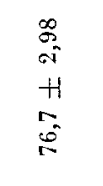 & $\begin{array}{l}8 \\
\infty \\
+1 \\
0 \\
\infty \\
0\end{array}$ \\
\hline & 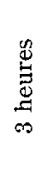 & $\begin{array}{l}\mathscr{8} \\
8 \\
o \\
+1 \\
0 \\
\vdots \\
=\end{array}$ & 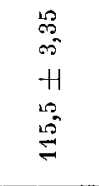 & $\begin{array}{l}\stackrel{P}{1} \\
\text { is } \\
H \\
0 \\
\stackrel{1}{5}\end{array}$ & $\begin{array}{l}10 \\
\infty \\
-i \\
H \\
0 \\
0 \\
50 \\
0\end{array}$ \\
\hline & 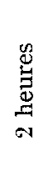 & 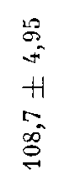 & $\begin{array}{l}\infty \\
\infty \\
\infty \\
+1 \\
-1 \\
\infty \\
\infty\end{array}$ & $\begin{array}{l}\overrightarrow{0} \\
0 \\
0 \\
+1 \\
+1 \\
\infty \\
\infty \\
\infty\end{array}$ & 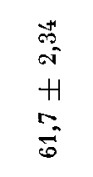 \\
\hline & $\begin{array}{l}\stackrel{\Xi}{\Xi} \\
\stackrel{\Xi}{\Xi} \\
=\end{array}$ & $\begin{array}{l}8 \\
5 \\
+1 \\
\vdots \\
\Xi\end{array}$ & 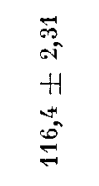 & \begin{tabular}{l} 
F \\
$\stackrel{-}{-1}$ \\
+1 \\
0 \\
\multirow{2}{*}{}
\end{tabular} & $\begin{array}{l}8 \\
\stackrel{8}{\circ} \\
+1 \\
0 \\
0 \\
0 \\
0\end{array}$ \\
\hline & 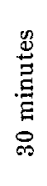 & 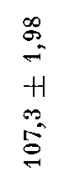 & $\begin{array}{l}\stackrel{8}{0^{2}} \\
+1 \\
\overrightarrow{9}\end{array}$ & 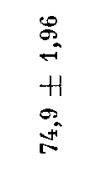 & 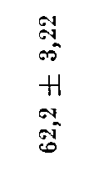 \\
\hline & 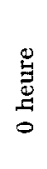 & $\begin{array}{l}5 \\
0 \\
0 \\
H \\
H \\
20 \\
0 \\
8 \\
0\end{array}$ & $\begin{array}{l}5 \\
5 \\
+1 \\
0 \\
5 \\
5\end{array}$ & 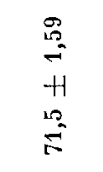 & $\begin{array}{l}8 \\
8 \\
o n \\
H \\
0 \\
0 \\
8\end{array}$ \\
\hline & 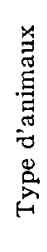 & 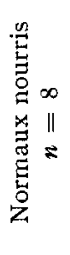 & 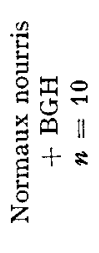 & 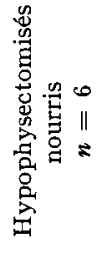 & 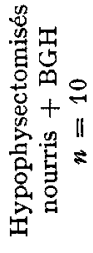 \\
\hline
\end{tabular}


A. VÉZINHET, J.-P. KHÉRIF, E. BOUTHIER

\begin{tabular}{|c|c|c|c|c|c|}
\hline \multirow{9}{*}{ 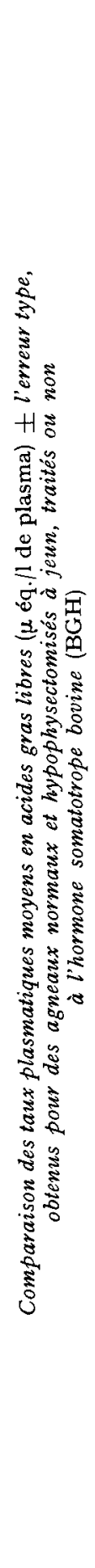 } & 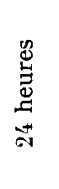 & 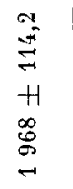 & 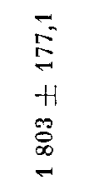 & 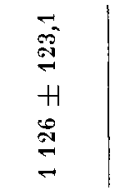 & 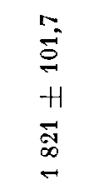 \\
\hline & 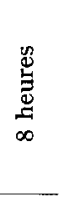 & 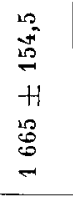 & 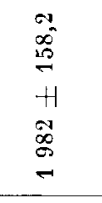 & 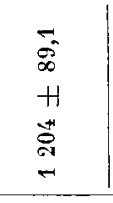 & 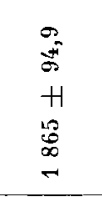 \\
\hline & 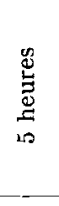 & 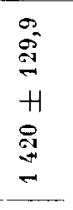 & 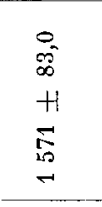 & 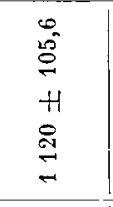 & 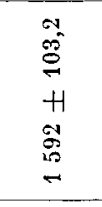 \\
\hline & 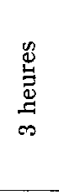 & $\begin{array}{l}5 \\
= \\
H \\
+ \\
=\end{array}$ & $\begin{array}{l}\text { Dे } \\
\stackrel{0}{9} \\
H \\
\text { 总 } \\
-\end{array}$ & 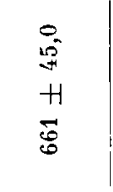 & 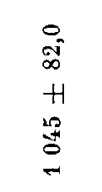 \\
\hline & 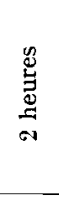 & 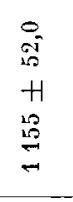 & 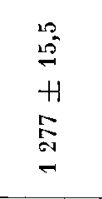 & 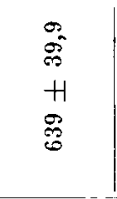 & 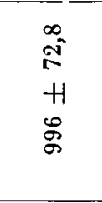 \\
\hline & 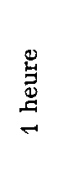 & $\begin{array}{l}\vec{i} \\
\stackrel{5}{8} \\
+1 \\
\stackrel{8}{5} \\
=\end{array}$ & 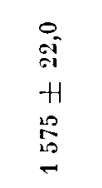 & 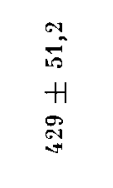 & 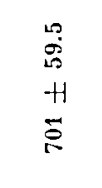 \\
\hline & 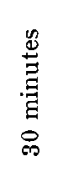 & 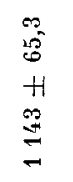 & $\begin{array}{l}\infty \\
0 \\
10 \\
H \\
1 \\
8 \\
8 \\
-1\end{array}$ & 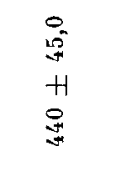 & $\begin{array}{l}\stackrel{+}{~} \\
\text { ه్ } \\
H \\
H \\
\mathscr{్}\end{array}$ \\
\hline & $\begin{array}{l}\stackrel{\Xi}{\Xi} \\
\stackrel{\Xi}{\Xi}\end{array}$ & $\begin{array}{l}0 \\
\stackrel{8}{8} \\
H \\
H \\
\text { 品 } \\
-\end{array}$ & $\begin{array}{l}0 \\
\text { it } \\
+1 \\
\text { Hon } \\
\text { S. } \\
-\end{array}$ & $\begin{array}{l}= \\
5 \\
H \\
\frac{1}{6}\end{array}$ & $\begin{array}{l}\overrightarrow{5} \\
\stackrel{5}{0} \\
H \\
\infty \\
0 \\
0 \\
0\end{array}$ \\
\hline & 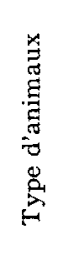 & 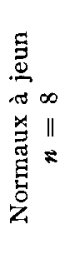 & 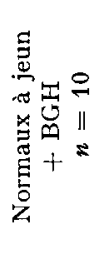 & 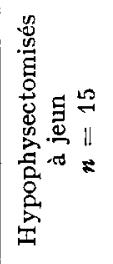 & 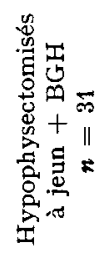 \\
\hline
\end{tabular}


BGH ET IIPOLYSE CHEZ L'AGNEAU

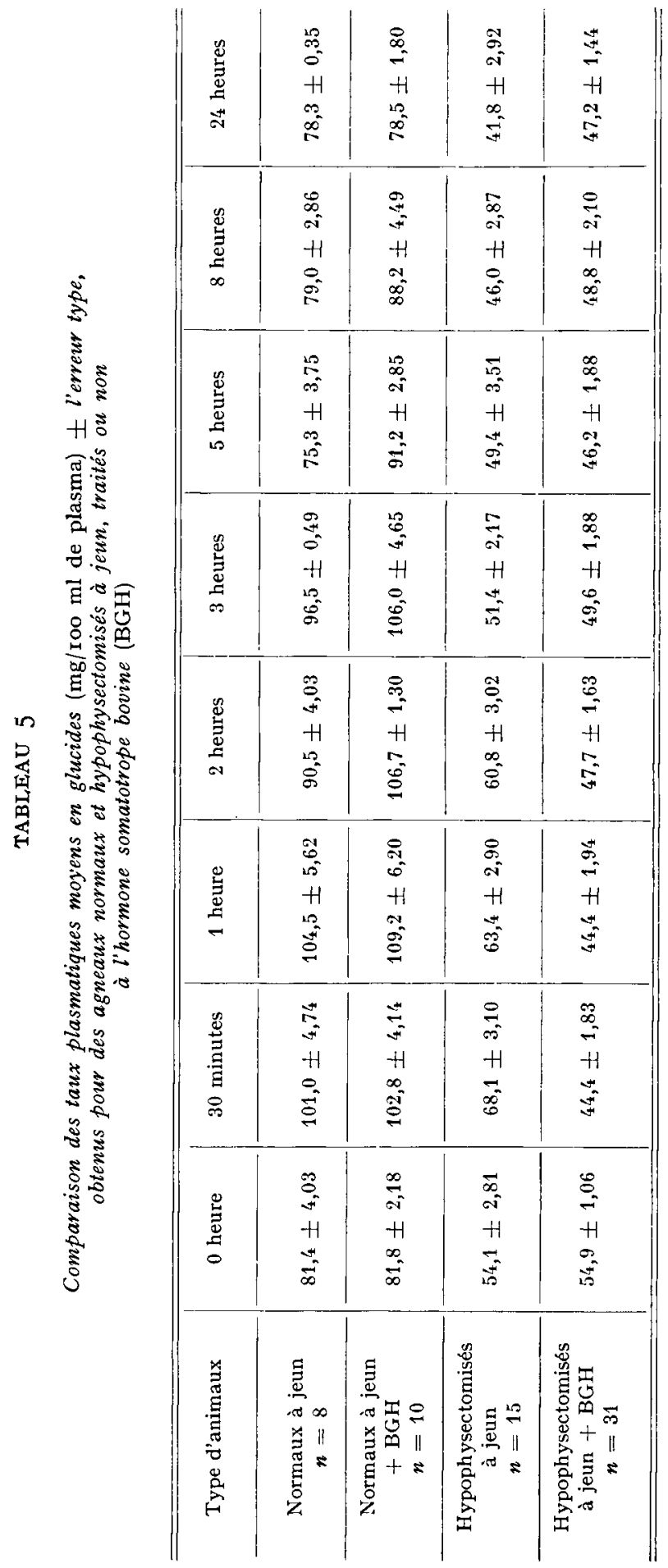




\section{Effet du jềne et du traitement BGH. Acides gras libres (tab1. 4)}

Pour des agneaux normaux, après un jeûne de 24 heures, les taux d'acides gras libres au temps o sont toujours plus élevés que pour les hypophysectomisés, environ le double (tabl. 4).

Avec ou sans traitement à la $\mathrm{BGH}$, on observe toujours, 30 minutes après le prélèvement du temps 0 , une chute des acides gras libres plasmatiques. Le retour à la normale s'effectue de façon différente suivant le type d'animaux. Il est très lent dans le cas des agneaux normaux sans traitement. Par contre il s'effectue dès la première heure pour les agneaux normaux traités à la BGH. Chez les hypophysectomisés la chute transitoire des acides gras libres 30 minutes après le traitement est également compensée beaucoup plus rapidement pour les agneaux traités à la BGH. Chez ce dernier type d'animaux la réponse lipolytique à la $\mathrm{BGH}$ est particulièrement nette; bien que partant de valeurs initiales en acides gras libres bien plus faibles que les agneaux normaux ils atteignent à 8 heures un maximum du même ordre de grandeur.

\section{Glycémie (tab1. 5)}

Chez les agneaux hypophysectomisés les glycémies sont nettement plus faibles que chez les normaux, le maintien de ces glycémies sous l'effet d'un jeûne prolongé semble difficile à réaliser notamment chez les hypophysectomisés sans traitement à la $\mathrm{BGH}$ pour lesquels on observe entre 30 minutes et 24 heures une diminution régulière des valeurs. Ce phénomène serait moins net chez les agneaux traités à la BGH. Pour les agneaux normaux à jeun traités ou non à la $\mathrm{BGH}$, on note après une élévation transitoire de la glycémie dans les trois premières heures, une diminution régulière, pour atteindre à 24 heures des valeurs très voisines de celles du temps 0 .

\section{DISCUSSION}

Le rôle lipolytique de l'hormone somatotrope apparaît nettement dans cette expérience. Chez les agneaux normaux nourris ad libitum il est décelable dès la première heure avec un maximum à la cinquième heure après le traitement hormonal. Ceci est en accord avec les résultats obtenus chez 1'Homme par FinGEL et al. (I959) avec de la GH humaine; ou par Altszuliter et al. (1968) chez le Chien avec de la GH bovine. Par contre WINKLER et al. (I962) toujours chez le Chien observaient avec de la $\mathrm{GH}$ bovine une réponse maximale dès la troisième heure.

Pour les agneaux hypophysectomisés nourris ad libitum il semble, dans cette expérience, que le démarrage de la réponse lipolytique à la $\mathrm{GH}$ soit moins rapide et moins intense que chez les normaux. La production globale d'acides gras libres dans les 24 heures qui suivent le traitement est également inférieure. Ce résultat rejoint celui obtenu chez le Lapin en réponse à de la GH porcine (VÉzInHET et BouthiEr, 1974). On pourrait donc penser que chez les agneaux hypophysectomisés le maintien des réserves adipeuses est assuré plus efficacement que chez les normaux. La lipolyse observée au temps o avant le traitement hormonal chez les agneaux à jeun va égale- 
ment dans ce sens. Ce résultat est en accord avec celui de Astwoop (I965), obtenu chez le Rat. La moindre réponse aux agents lipolytiques chez des agneaux nourris et la meilleure résistance au jeûne pourraient donc contribuer à la surcharge adipeuse observée chez les agneaux hypophysectomisés maintenus longuement en survie (VÉZINHET, r973).

Les agneaux hypophysectomisés à jeun traités à l'hormone somatotrope manifestent par contre une décharge importante d'acides gras libres dès la première heure après le traitement avec un maximum à la huitième heure. Dans le cas d'une mise à jeun préalable au traitement hormonal la production globale d'acides gras libres sur 24 heures est nettement supérieure à celle notée chez les agneaux normaux maintenus dans les mêmes conditions alimentaires. Ce résultat à l'opposé de celui observé pour des agneaux nourris est en accord avec celui de KNOBIL et GREEP (I959) obtenu chez le Singe Rhésus, pour lequel une simple injection de GH de singe faisait accroitre le taux plasmatique des acides gras libres chez l'hypophysectomisé d'autant plus nettement que celui-ci était à jeun.

Un autre point mérite d'être signalé, il concerne l'abaissement très net des taux plasmatiques en acides gras libres, chez les agneaux à jeun normaux et hypophysectomisés, 30 minutes après le traitement hormonal. Certains auteurs comme SIREK et al. (I964), WEIL (I965) avaient observé cette chute transitoire des acides gras libres aussitôt après l'injection de l'hormone, l'interprétation de ce phénomène s'avérait délicate.

Le fait que nous ayons observé la même chute chez les agneaux à jeun sans traitement hormonal et que nous ne l'ayons jamais mis en évidence chez les agneaux nourris, laisse penser qu'il pourrait s'agir d'un effet "stress " consécutif au premier prélèvement de sang. Dans ce cas il faudrait admettre que les agneaux à jeun sont plus sensibles à cet effet que les agneaux nourris. En outre l'effet "stress " se traduit généralement par un accroissement des taux plasmatiques en acides gras libres consécutif à une libération massive de ces métabolites par le tissu adipeux. Il s'agirait donc ici d'un phénomène de nature différente qui pourrait être au contraire une prise en charge accrue des acides gras libres par les tissus utilisateurs en réponse au "stress".

L,es résultats obtenus avec les glycémies n'appellent pas de notre part de commentaires particuliers, si ce n'est les valeurs nettement inférieures observées dans tous les cas chez les hypophysectomisés par rapport aux agneaux normaux. Dans le cas du jêtne on obtient même des valeurs très faibles qui se trađuisent parfois chez l'animal par des troubles de type hypoglycémiant.

En conclusion il apparaît que la $\mathrm{GH}$ outre son rôle somatotrope pourrait, par son effet lipolytique, contribuer à l'utilisation des graisses de réserve chez le Mouton ; elle appartiendrait ainsi aux substances lipolytiques qui concourrent, par la libération des acides gras à partir du tissu adipeux, à la couverture des besoins énergétiques de l'animal. Le problème de savoir comment la GH exerce son effet lipolytique chez 1'Agneau reste cependant posé si l'on considère le peu d'effet qu'ont le régime alimentaire, l'âge, la race ou le sexe, sur les variations en GH plasmatiques (TRENKLE, I97I, IRVIN et TRENKLE, I97I) ou en GH hypophysaire (CHARRIER, I973). 


\section{REMERCIEMEN'TS}

Ce travail a été en partie réalisé dans le cadre d'une action complémentaire coordonnée "Biologie de la Reproduction et du Développement " de la Délégation Générale à la Recherche Scientifique et Technique, contrat no 73-7-1654.

\section{SUMMARY}

\section{LIPOLYTIC EFFECT IN VIVO OF SOMATOTROPIC HORMONE IN NORMAL AND HYPOPHYSECTOMIZED I AMBS FASTED OR NOT}

The lipolytic effect of a strong bovine somatotropic hormone treatment (BGH) on the normal and hypophysectomized lamb is studied on fully fed animals or after a good 24-hour fast. Blood sugar development is followed in the same conditions.

- Lipolysis induced by BGH seems to be stronger in normal than in hypophysectomized lambs when they are fully fed.

- Free fatty acid plasma content is lower in hypophysectomized than in normal lambs after 24 hours of fasting.

- These two observations indicate that the maintenance of adipose reserves is more efficiently insured in hypophysectomized than in normal lambs.

- On the other hand, Iipolytic response to BGH is particularly strong in hypophysectomized lambs when fasted.

\section{RÉFÉRENCES BIBLIOGRAPHIQUES}

Altszuller N., Rathgeb I., Winkler 13. N., DE Bodo R. C., rg68. The effects of growth hormone on carbohydrate and lipid metabolism in the dog. Ann. N. Y. Acad. Sci., 148, 44I-458.

Astwood E. B., I965. The pituitary gland and the mobilization of fat, Handbook of physiology, Adipose tissue, American physiological society. Eds : RenOLD A. E., CAHILl G. F., American physiological society, Washington; sect. 5, 529-532.

Charrier J., i973. Évolution foetale et postnatale du contenu en hormone de croissance de l'hypophyse ovine. Ann. Biol. anim. Bioch. Biophys., 13, 155-163.

Chrétien M., Lis M., Bertagna X., Gilardeau C., 1973. Les hormones lipolytiques hypophy. saires. In hormones et régulations métaboliques. Masson Paris, 249-272.

Dole V. P., Mernertz H., I960. Microdetermination of long chain fatty acids in plasma and tissues. J. biol. chem., 235, 2595-2599.

Engel H. R., Bergenstal D. M., Nixon W. E., Patton J. A., I959. Effect of human growth hormone on unesterified fatty acid and plasma amino acid nitrogen in man. Proc. Soc. exp. Biol. Med., 100, 699 .

Irvin R., Trenkle A., I97i. Influences of age, breed and sex on plasma hormones in cattle. J. Anim. Sci., 32, 292-295.

KNobil E., Greep R. O., I959. The physiology of growth hormone with particular references to its action in the rhesus Monkey and the "species specificity" problem. Recent. Prog. Horm. Res., 15, $\mathrm{I}-58$.

Machlin L. J., Takahashi Y., Horino M., Hertelendy F., Gordon R. S., Kipnis D., I968. Regulation of growth hormone secretion in non primate species. In Growth hormone. Eds. A. PECILE and E. E. Müller. Excerpta Medica Foundation. International Congress Series n ${ }^{\circ}$ I 58, 292-305.

Manns J. G., Boda J. M., I965. Effects of ovine growth hormone and prolactin on blood glucose, serum insulin, plasma non esterified fatty acids and amino nitrogen in sheep. Endocrinology, 76, x I09I I I 5 . 
Nelson N., I944. A photometric adaptation of the Somogyi method for the determination of glucose. J. biol. chem., 153, 375-380.

Raben M. S., Hollenberg C. H., I958. Effect of growth hormone on fatty acids. J. Clin. Invest., 37, $922-926$.

Sirek O. V., Sirek A., Przybylska K., Doolan H., Niki A., ig67. Plasma free fatty acids concentrations in Houssay dogs following a single injection of growth hormone. Endocrinology, 81, 395-398.

TRENKLE A., 1971, Effect of diet upon levels of plasma growth hormone in sheep. J. Anim. Sci., 32, III-II 4 .

Vézinhet A., I968. Effet de l'hypophysectomie sur la croissance pondérale de l'Agneau. C. R. Acad. Sc., 266, 388.39o.

Vézinhet A., I973. Influence de l'hypophysectomie et de traitements à l'hormone somatotrope bovine sur la croissance relative de l'Agneau. Ann. Biol. anim. Bioch. Biophys., 13, 5I-73.

Vézinhet A., Bouthier E., I974. Effet lipolytique in vivo et in vitro de l'hormone somatotrope porcine sur le tissu adipeux de lapins normaux et hypophysectomisés. C. R. Acad. Sc., 278, 3I I-3I4.

WEIL R., I965. Pituitary GH and intermediary metabolism. I. The hormonal effect on the metabolism of fat and carbohydrate. Acta entocr., suppl. 98, 9 I p.

Winkler B. N., Steele R., Altszuller N., Dunn A., De Bodo R. C., I962. Effects of growth hormone on FFA metabolism. Fed. Proc., 21, 198. 